\title{
Atomic Scale Characterization of LSFMO Thin Films Using Aberration Corrected STEM
}

\author{
M. Gu, ${ }^{*}$ Y. Takamura* and N. D. Browning******
}

* Department of Chemical Engineering and Materials Science, University of California-Davis, One Shields Avenue, Davis, CA95616

** Department of Molecular \& Cellular Biology, University of California-Davis, One Shields Avenue, Davis, CA95616

*** Condensed Matter and Materials Division, Lawrence Livermore National Laboratory, P.O. Box 808; Livermore, CA 94550

Superlattices consisting of ferromagnetic metal $\mathrm{La}_{(1-\mathrm{x})} \mathrm{Sr}_{\mathrm{x}} \mathrm{MnO}_{3}(\mathrm{LSMO})$ and the antiferromagnetic insulator $\mathrm{La}_{(1-\mathrm{x})} \mathrm{Sr}_{\mathrm{x}} \mathrm{FeO}_{3}$ (LSFO) have attracted a lot of scientific interest for their potential application as next generation data storage materials. [1-3] In this work, epitaxial thin films of the LSMO/LSFO solid solution, $\mathrm{La}_{0.3} \mathrm{Sr}_{0.7} \mathrm{Fe}_{0.5} \mathrm{Mn}_{0.5} \mathrm{O}_{3}$ (LSFMO), were grown on single crystal $\mathrm{LaAlO}_{3}(\mathrm{LAO})$ substrates by pulsed laser deposition. These samples are chemically equivalent to the LSMO/LSFO superlattices but without the presence of the high density of interfaces. Previous studies show that Fe doping in LSMO bulk crystal suppresses on the conduction and ferromagnetism and changes the density of states near Fermi level. [4]

We performed a detailed characterization of the atomic structure of LSFMO thin films by combining dark field and bright field images acquired simultaneously by an aberration-corrected scanning transmission electron microscopy (STEM). All dark field images are taken using a high angle annular dark field (HAADF) detector with an inner angle of 100 mrads compared to 24mrads convergence angle. These images identify an interface region formed due to cation interdiffusion at the film/substrate interface. As shown in FIG.1 and FIG.2, the contrast in Z-contrast image is mainly proportional to the average atomic mass, therefore, comparatively the LAO region appears very bright, the thin film region appears very dark due to lower average $\mathrm{Z}$ number and the interface region appears with intermediate contrast due to cation interdiffusion between substrate and thin film. The three images in FIG.3 were obtained by focusing on three peaks of the histogram in FIG.2 which clearly identifies the brightest part of the image to the substrate, the intermediate contrast to the interface region, and the dark contrast to the thin film region. The observed cation interdiffusion directly affects the Fe and Mn valence states within the interface region and the vicinity of the interface. Electron energy loss spectroscopy (EELS) mapping the profile of the Fe and Mn valence states across the substrate, the interface region, and the thin film in relationship to the cation diffusion will also be presented.

References

[1] M. Varela, et al, Phys. Rev. B, 79 (2009) 085117

[2] M. Izumi, et al, Materials Science and Engineering B84 (2001) 53

[3] Y. Takamura, et al., Phys. Rev. B, 80(2009) 180417(R)

[4] A V Deshmukh, et al, J. Phys. D: Appl. Phys. 42 (2009) 185410

[5] This research was supported by the NSF(contract No. DMR-747896) and DOE (contract No.

DE-FG02-03ER46057). 


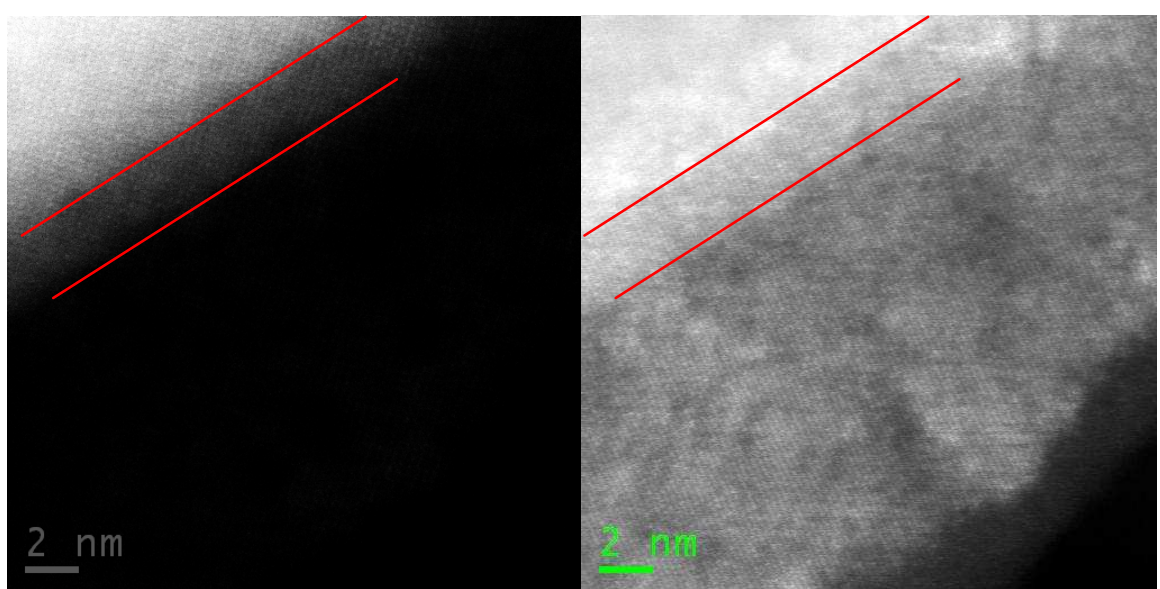

FIG. 1. Dark field (left) and bright field image (right) of LSFMO on LAO taken simultaneously, showing the single crystallinity of the thin film. The region between the red lines corresponds to interface diffusion region.
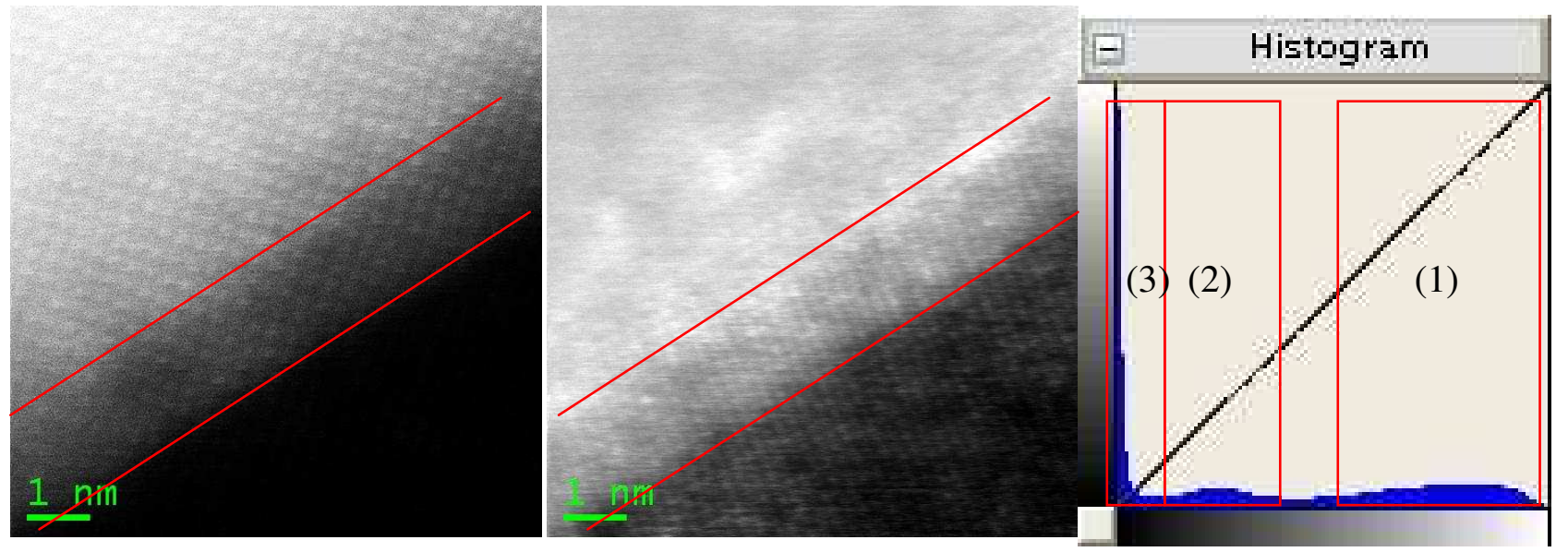

FIG. 2. Dark field image(left), bright field image(middle) and the histogram of the dark field image(right). Red line region signifies the interface region.

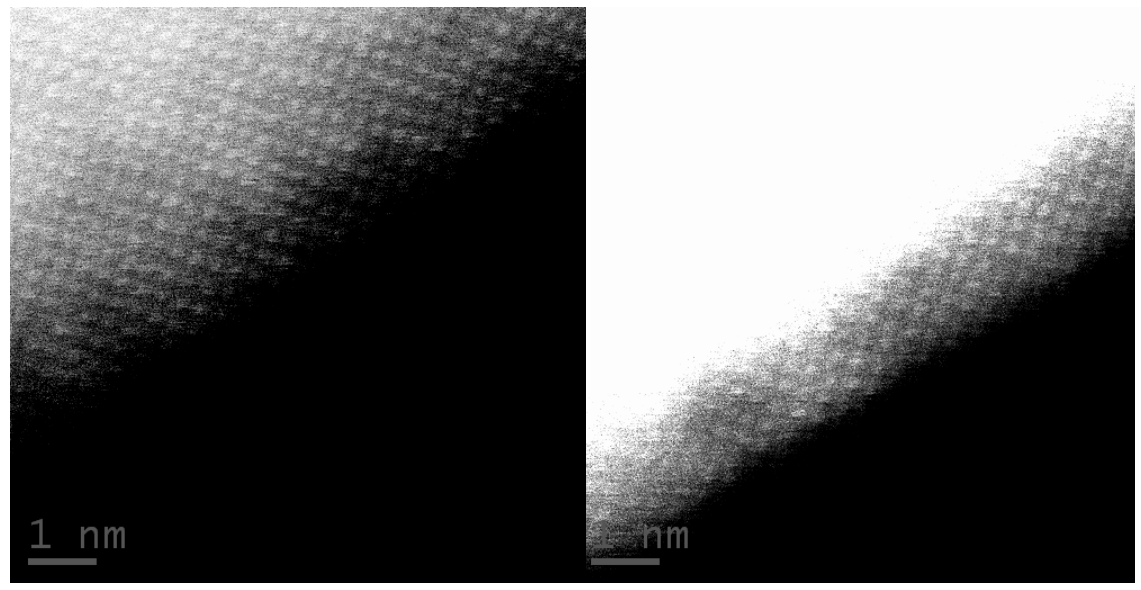

FIG. 3. Images of the substrate (left), interface (middle), thin film (right) region obtained by focusing on regions (1), (2), (3) of the histogram in Figure 2, respectively. 